\title{
A new Approach for Scaling Up from a Small Cold to a Large Hot Bed for Biomass Conversion in a Bubbling Fluidized Bed Reactor
}

\author{
Cornelius E. Agu ${ }^{1}$, Rajan K. Thapa ${ }^{1}$, Christoph Pfeifer ${ }^{2}$, Lars-Andre Tokheim ${ }^{1}$, Britt M.E. Moldestad ${ }^{1}$ \\ ${ }^{1}$ Department of Process, Energy and Environmental Technology, University of South-Eastern Norway, 3918 \\ Porsgrunn, Norway, \{Cornelius.e.agu, rajan.k.thapa, Lars.A.Tokheim, britt.moldestad\}@usn.no \\ ${ }^{1}$ Department of Material Sciences and Process Engineering, University of Natural Resources and Life Sciences, 1190 \\ Vienna, Austria, christoph.pfeifer@boku.ac.at
}

\begin{abstract}
Bubbling fluidized beds are simple and attractive means of achieving efficient conversion of biomass if particle segregation and the associated effects are minimized. To improve the knowledge of fluidized bed reactor design, this paper compares the behavior of a hot bed containing a certain amount of biomass with the behavior in a cold bed having the same biomass loads and particle properties. An approach for scaling up a cold bed to a large hot bed for the same volume fraction of biomass is introduced. The proposed scheme uses the bed expansion ratio as an output from the cold bed. This approach provides an accurate means of attaining dynamic similarity in bubbling behavior between two different beds without constraining the fluid and particle properties as well as the bed height.
\end{abstract}

Keywords: biomass, scale up, bed expansion, CPFD, bubbling fluidized bed

\section{Introduction}

To enhance the understanding of fluidized bed behavior for reactor designs and scale up, this paper compares the bubbling behavior in a hot fluidized bed containing biomass with that observed in Agu et al. (2019a) under cold flow condition using the same bed material and biomass properties. In the literature (Agu et al., 2019a), the mixing and segregation pattern of wood chips and pellets of a wide density difference but similar volumeequivalent spherical particle diameter was studied using electrical capacitance tomography (ECT).

Understanding how the cold bed behavior is related to the behavior in an active hot bed is often a challenge in modelling and scaling up. The traditional scaling up approaches (Glicksman et al., 1993; Glicksman, 1988; Glicksman, 1984; Romero and Johanson, 1962) that have been used in many studies (Kraft et al., 2018; Biglari et al., 2016; Thapa and Halvorsen, 2014) do not consider the influence of feedstock and volatiles (i.e. biomass) on the bubbling behavior. As shown in different studies (Agu et al, 2019a; Fotovat et al, 2015a), the amount of biomass in a bed affects the bubble diameter, bubble frequency and bed expansion, and also causes a delay in slug flows. In an attempt to keep the ratios of diameter and density of fuel particles to those of a bed material constant, the two beds will have different fluid and particles properties. Different scaling up approaches accounting for intrinsic behavior in a bed have also been demonstrated. On the account of segregation effects, a scaling approach based on the biomass residence time distribution was reported in (Zhang and $\mathrm{Xu}, 2015$ ). Scaling up a bed based on the segregation effects on the thermochemical conversion of biomass can also be achieved by using the relative time scales (Damköhler numbers) between the segregation and devolatilization of the fuel particles (Salatino and Solimene, 2017). Moreover, a bubbling fluidized bed can also be scaled up by keeping at least one of the bubble properties constant (Agu et al, 2019b).

In this study, a new approach for scaling a bubbling bed behavior from a cold small to a hot large bed is introduced based on a combination of Horio et al (1986) scaling law and correlations for minimum fluidization velocity and bed expansion ratio. The scaling approach introduced allows the same properties of biomass as well as the same bed material type to be used in both beds. For the scaled bed, the Wen and Yu (1966a) correlation is used to determine the particle size while the model for bed expansion ratio (Agu et al., 2019c) is used to determine the aspect ratio (bed height to diameter) owing to the fact that bubble growth and bed expansion depend on the bed aspect ratio. The scaled bed aspect ratio is also verified by simulation of the two different beds using the Barracuda software based on the CPFD (computational particle-fluid dynamics) model.

\section{Modelling and scaling up}

To scale up fluidized bed behavior from a small to a larger bed diameter, scaled and transferrable information from the smaller (reference) bed is required. In a hot flow bed, temperature and pressure are easier to measure. Temperature is not a product of bubble flows but the distribution of temperature as well as the pressure fluctuations can be linked to the bubbling behavior. However, modelling of temperature distribution and pressure fluctuation is a complex task. 
In a cold bed, different properties can easily be measured, including bubble diameter, bubble frequency and bed expansion. Different correlations are also available in literature for prediction of the different bubble properties for a bed of single particle type, e.g. sand particles. Based on the results reported in Agu et al (2019a) for binary mixtures of sand and biomass, none of the available models (Choi et al, 1988; Darton et al, 1977; Mori and Wen, 1975) for bubble diameter can predict the bed behavior with a good accuracy. The bed expansion predicted using different models (Agu et al, 2019c; Hepbasli, 1998; Werther, 1978) at $U_{0} / U_{m f s}=2$ show similar trends as the experimental data. Numerically, the Agu et al (2019c) model agrees very well with the data from the pellet beds while for the wood chip beds, a good agreement was obtained when the segregated layer at the surface of the bed is excluded in the total bed height measured. The bed expansion model as proposed by Agu et al (2019c) is described below:

$$
\begin{aligned}
& \Delta e=\left[1-0.103\left(U_{0}-U_{m f}\right)^{-0.362}\left(\frac{\bar{d}_{b}}{D}\right)\right]^{-1}-1 \\
& \bar{d}_{b} / D=0.848\left(\frac{U_{0}}{D}\right)^{0.66}\left(1-c\left(\frac{U_{0}}{U_{m f}}\right)^{a-1}\right)^{0.66} \\
& A r>400 \text { and } U_{0} / U_{m f}>c^{1 /(1-a)}
\end{aligned}
$$

where $\Delta e=\left(H_{f}-H_{m f}\right) / H_{m f}$ is the bed expansion and $\bar{d}_{b} / D$ is the bubble to bed diameter ratio averaged over the bed height. $H_{m f}$ and $H_{f}$ are bed heights at the mixture minimum fluidization velocity $U_{m f}$ and at the operating gas velocity $U_{0}$, respectively. Eq. (1) is semiempirical and was developed based on the mass balance in the solid and gas phases. By using the particledependent average bubble diameter shown in Eq. (2) as proposed by Agu et al (2018), Eq. (1) predicts the behavior in beds with different particle properties. $A r$ in Eq. (2) is the Archimedes number, and the equation is applicable for large particles where $A r>400$ and for gas velocities $U_{0}$ such that $U_{0} / U_{m f}>c^{1 /(1-a)}$ which corresponds to the minimum bubbling condition of the bed, where $a$ and $c$ can be obtained as described in the literature (Agu et al, 2018).

For a binary mixture of sand and biomass particles, the mean particle diameter $d_{m}$, density $\rho_{m}$ and sphericity $\varphi_{m}$ used for evaluation of $\bar{d}_{b} / D$ can be obtained from the following, where the subscripts " $\mathrm{m}$ ", "b" and "s" denote mixture, biomass and sand, respectively, and $y_{b}$ is the biomass volume fraction in the binary mixture:

$$
\begin{aligned}
& d_{m}=\left[\frac{y_{b}}{\varphi_{b} d_{b}}+\frac{\left(1-y_{b}\right)}{\varphi_{s} d_{s}}\right]^{-1} \\
& d_{p, s p h, m}=\left[\frac{y_{b}}{d_{b}}+\frac{\left(1-y_{b}\right)}{d_{s}}\right]^{-1} \\
& \rho_{m}=y_{b} \rho_{b}+\left(1-y_{b}\right) \rho_{s} \\
& \varphi_{m}=d_{m} / d_{p, s p h, m}
\end{aligned}
$$

\section{Results and discussion}

Figure 1 compares the predictions of the bed expansion using Eq. (1) with the experimental data obtained at different gas velocities and biomass loads. For the bed of pellets, the mean bed particle properties are based on the total amount of biomass charged in the bed. However, in the wood chip bed, the computation of the mean particle properties is based on $50 \%$ of the total biomass charged since approximately $50 \%$ of the biomass load lies above the bed surface, preventing the bubble passage (Agu et al, 2019a). As can be seen in Figure 1(a), the model results agree very well with the measured data from the wood chip beds, particular with $y_{b}=0.2$. For the pellet beds, the model prediction accuracy increases with increasing gas velocity. The inaccuracy in predicting the bed expansion can be associated with the strong interactions between the biomass and sand particles at lower gas velocities. With low gas velocity, a higher amount of sand particles are in the interstices between the biomass particles, thus reducing the bed voidage and expansion. Increasing the gas velocities increases the inter-particle spacing, thereby enhancing the bed expansion.

\subsection{Scale up from cold small to hot large bed}

To attain a dynamic similarity in the flow of bubbles between a cold small bed and a hot larger bed based on the same biomass properties and volume fraction, the Horio et al. (1986) scaling law as described below can be applied.

$$
\frac{U_{0}-U_{m f}}{\sqrt{g D}}, \frac{U_{m f}}{\sqrt{g D}}
$$

The Horio et al (1986) law does not impose a restriction on the bed height and on the solid to fluid density ratio. In addition, Glicksman (1988) showed that (7) is equivalent to the scaling law proposed for the viscous limit set for two different beds of the same geometry and negligible solid to fluid density ratio. Implementation of Eq. (7) in this study for scaling up the biomass-sand bed behavior without considering the height and density ratios is based on the fact that bubble growth in a fluidized bed depends on the particle size, fluid properties and bed aspect ratio (Agu et al, 2019b). For larger particles or less viscous flow, the bubble growth rate with an increase in gas velocity is high. The bubble size increases with an increase in the bed aspect ratio, particularly for small particle sizes. From (7), 


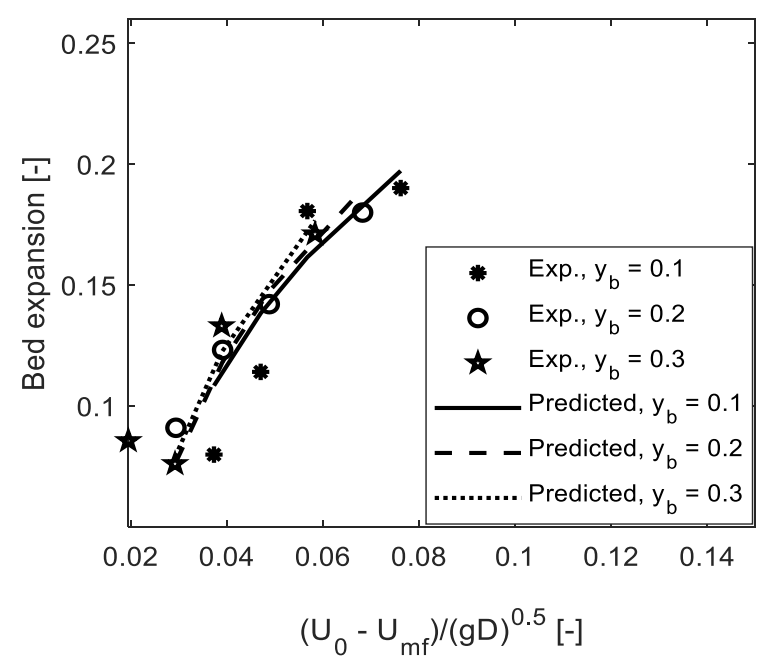

(a)

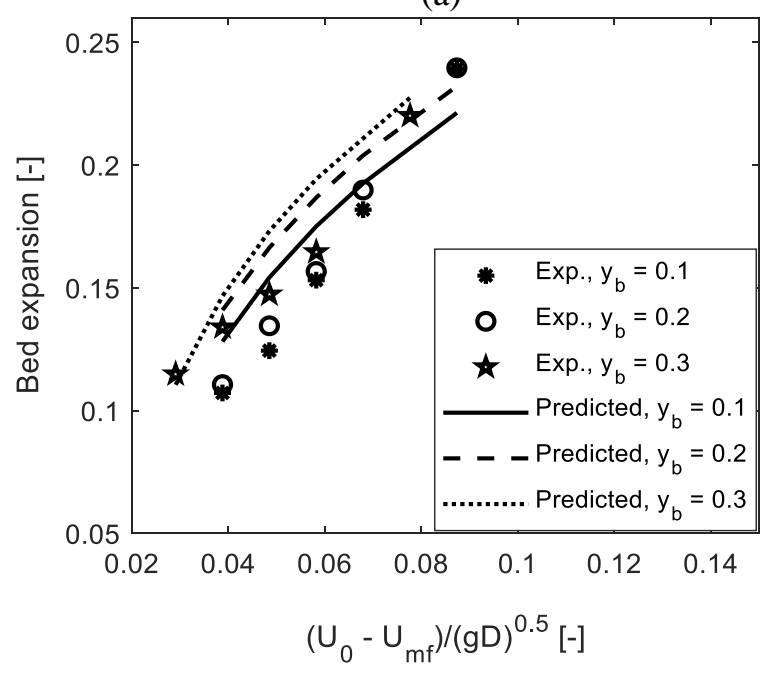

(b)

Figure 1. Bed expansion ratio, comparing the predicted results based on Eq. (1) with the experimental data obtained in the cold bed of diameter $10.4 \mathrm{~cm}$ at different biomass loads and air velocities as described in Agu et al. (2019a); (a) wood chips (b) wood pellets.

$$
U_{m f 2}=\sqrt{r} U_{m f 1}
$$

where $r=D_{2} / D_{1}$ is the size ratio between the large bed diameter $D_{2}$ and the small bed diameter $D_{1}$, and $U_{m f 2}$ and $U_{m f 1}$ are the corresponding minimum fluidization velocity of the particles in the respective beds. Eq. (8) indicates that particles in the larger bed will have a larger particle size since $U_{m f 2}>U_{m f 1}$. The particle size for a given value of $U_{m f}$ can be obtained from Eq. (9) as proposed by Wen and Yu (1966a).

$$
\begin{aligned}
& \frac{\rho_{g} U_{m f} d_{s}}{\mu_{g}}=\left[-33.67+\sqrt{\left((33.67)^{2}+0.0408 A r\right)}\right] ; \\
& A r=\frac{d_{s}^{3} \rho_{g}\left(\rho_{s}-\rho_{g}\right) g}{\mu_{g}^{2}}
\end{aligned}
$$

If the aspect ratio $h_{0} / D$ is the same for both beds, the resulting bed height for the larger bed may be too high for industrial application; in addition, the bubble size at the same $\left(U_{0}-U_{m f}\right) / \sqrt{g D}$ value may be larger. Therefore, for successful application of the Horio et al (1986) scaling law, an output from the large bed that must be correctly matched with that from the smaller bed is required. For the binary mixture of biomass and sand particles, the bed expansion ratio is the output applied in the scaling approach introduced in this study. The bed expansion of the scaled hot larger bed can be simulated from Eq. (1). By comparing the simulated result with the measured data from the cold small bed at the same biomass volume fraction, the appropriate bed aspect ratio can be determined. Since Eq. (1) accurately predicts the behavior in the cold bed of diameter 10.4 $\mathrm{cm}$ containing $20 \%$ wood chips, this bed is used as the reference here.

Figure 2 compares the bed expansion predicted for the scaled hot large bed with those measured in the cold small bed at different biomass loads. The new bed aspect ratio is evaluated as $h_{0 *} / D_{*}=2.7$, where $h_{0 *}=50 \mathrm{~cm}$ is the initial height of the reference bed (cold bed) and $D_{*}=18.5 \mathrm{~cm}$ is the characteristic bed diameter obtained by fitting (1) at the hot bed condition with the data obtained from the cold bed at $y_{b}=0.2$. As can be seen, there is a good agreement between the simulated results and the experimental values from the bed with the 20 vol.\% wood chips. The similarity also observed for other wood chip beds and those of pellets confirms the validity of the scaling approach.

Different dimensionless groups associated with the two beds based on the bed materials are compared in Table 1 . In addition to the Froude numbers, $U_{m f}{ }^{2} /$ $(g D)$, the particle Reynolds numbers, $\rho_{g} U_{m f} d_{s} / \mu_{g}$ and Archimedes numbers, $A r=d_{s}^{3} \rho_{g}\left(\rho_{s}-\rho_{g}\right) g / \mu_{g}^{2}$ are closely matched. To achieve the same $h_{0} / d_{s}$ ratio, the aspect ratio of the larger bed has to be reduced to $h_{0} / D=1.47$, which is possible if only the pure bed material is in the bed. However, by considering that the bed expansion ratio decreases with an amount of biomass in the bed, the height of the large bed needs to be as high as possible to achieve a similar behavior to that in the smaller bed diameter.

\subsection{Verifying the scaled bed aspect ratio}

To verify the aspect ratio of the scaled large bed, the two different beds (cold at ambient and hot at $800{ }^{\circ} \mathrm{C}$ ), each containing 20 vol. $\%$ wood chips were simulated using Barracuda VR software. Barracuda is a commercially developed platform for implementing the computational particle-fluid dynamics (CPFD) scheme. CPFD is based on the multiphase-particle-in-cell (MP-PIC) concept introduced by Andrew and O'Rourke (1996). In the CPFD scheme, the Euler-Lagrangian modelling approach is applied for fluid volume and particle tracking in gas-solid systems. With the MP-PIC concept, the computational particle represents a large number of particles with similar properties. 


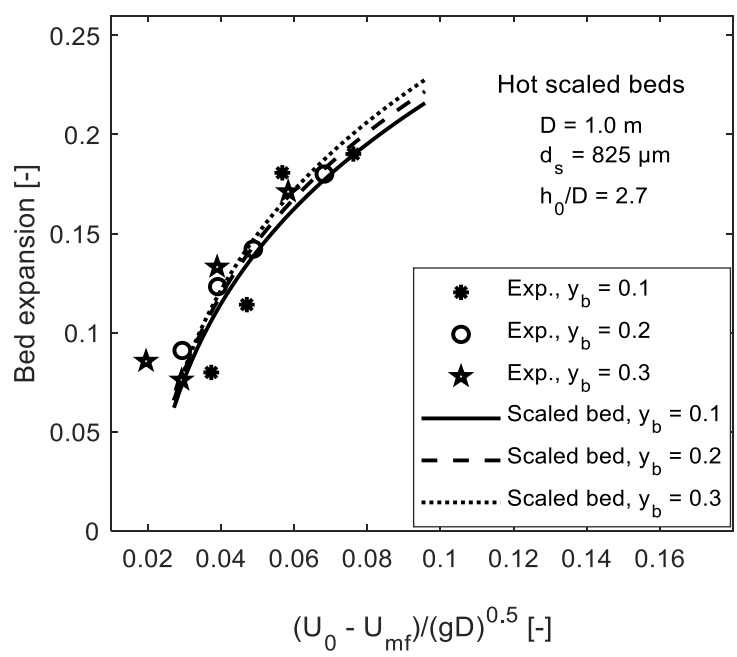

(a)

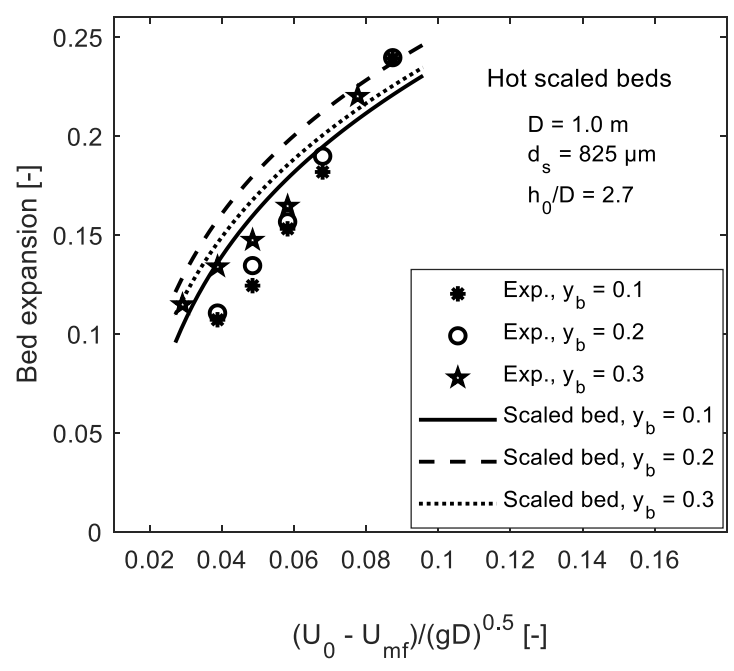

(b)

Figure 2. Bed expansion, comparing the bubbling behavior in the experimental small cold bed (diameter $=10.4 \mathrm{~cm}$, and $293 \mu \mathrm{m}$ sand particles) with that in the hot scaled bed of diameter $100 \mathrm{~cm}$ containing $827 \mu \mathrm{m}$ sand particles at different biomass loads of (a) wood chips and (b) wood pellets.

The grouping of particles in the CPFD code makes the simulation faster, thereby increasing its application to industrial systems. Detailed descriptions of the CPFD model and its numerical scheme can be found in Chen et al (2013). The Barracuda software has been successfully used in different studies including investigation of fluid-particle behavior in a binary mixture of bed material and biomass particles (Fotovat et al, 2015b) and classification of particles of different sizes and densities (Chladek et al, 2018).

In implementing the CPFD model, the particle properties were the same for both biomass and sand particles as those used in the cold bed experiment (Agu et al, 2019a). Both beds ( $D=10.4 \mathrm{~cm}$ and $D=100$ $\mathrm{cm}$ ) contain 20 vol\% wood chips for each air velocity.
Table 1. Comparison of the dimensionless numbers between the small cold and the large hot beds.

\begin{tabular}{|c|l|l|l|}
\hline Parameter & Unit & $\begin{array}{l}\text { Small bed } \\
\text { with 20 vol.\% } \\
\text { biomass }\end{array}$ & $\begin{array}{l}\text { Large Bed } \\
\text { with 20 vol.\% } \\
\text { biomass }\end{array}$ \\
\hline$T$ & ${ }^{\circ} \mathrm{C}$ & 25 & 800 \\
\hline$p$ & $\mathrm{~atm}$ & 1 & 1 \\
\hline$D$ & $\mathrm{~m}$ & 0.104 & 1.0 \\
\hline$\rho_{g}$ & $\mathrm{~kg} / \mathrm{m}^{3}$ & 1.18 & 0.33 \\
\hline$\mu_{g}$ & $\mathrm{~Pa} . \mathrm{s}$ & $1.8 \times 10^{-5}$ & $4.3 \times 10^{-5}$ \\
\hline$d_{s}$ & $\mu \mathrm{m}$ & 293 & 827 \\
\hline$\rho_{s}$ & $\mathrm{~kg} / \mathrm{m}^{3}$ & 2650 & 2650 \\
\hline$U_{m f}$ & $\mathrm{~m} / \mathrm{s}$ & 0.079 & 0.243 \\
\hline$U_{m f} /(g D)$ & - & $6 \times 10^{-3}$ & $6 \times 10^{-3}$ \\
\hline$\rho_{g} U_{m f} d_{s}$ & - & 1.54 & 1.53 \\
\hline$\mu_{g}$ & & & 2600 \\
\hline$A r$ & - & 2500 & 2.7 \\
\hline$h_{0} / D$ & - & 4.8 & 3300 \\
\hline$h_{0} / d_{s}$ & - & 1800 & \\
\hline
\end{tabular}

The airflow rate at the bottom of the column was assumed uniform across the bed. There are different drag models in the Barracuda library. As the Wen and $\mathrm{Yu}$ (1966b) drag model has been used in many applications with good results, this model was also used for all simulations in this study. The default model settings, as given in the Barracuda 17.05 version, were applied.

The geometries are divided into uniform grids. The grid size for the small bed is $13 \times 13 \times 12.2 \mathrm{~mm}$ while that of the larger bed is 9.6 times larger. Relatively coarse grid sizes were used to accommodate the biomass particles (6.87 mm diameter) within each computational cell since in Barracuda it is difficult to capture particles larger than the grid size. Each bed was initialized as a uniform mixture containing $20 \%$ wood and $80 \%$ sand particles over the bed height. The biomass and sand particles were set as two different particle species to track the individual particle motion and location along the bed. The bottom part of the bed was set as the velocity flow boundary and the top as the pressure boundary. Four different superficial gas velocities were applied in each bed. The simulations were run for $20 \mathrm{~s}$ with an initial time step of $0.001 \mathrm{~s}$. The fluid pressure and solid volume fraction at two different positions along the bed axis were used for further analysis.

Figure 3 ( $a$ and $b$ ) compares the distribution of the solid species (sand and biomass particles) between the two different beds from the initial well mixed state to the final state. For the same biomass volume fraction, the number of biomass particles per unit length is 
proportional to the cross-sectional area of the bed. As a result, the scale on the colour bar reflects a higher biomass concentration in the hot bed compared to the cold bed, which is 100 times smaller in cross-sectional area. At the final state, the results show that particle distributions are similar in both beds; most of the biomass particles moved to the bed surface as similarly observed in the experiments. The pressure value at the two indicated positions in the different beds were used as the basis for Figure 3 (c), which shows the variation of pressure drop normalized with the mean value within the last $5 \mathrm{~s}$ of the simulations. As can be seen, the fluctuation of pressure drop is similar in both beds, indicating that bubbles rise at approximately the same frequency in the beds. The higher fluctuation amplitude in the hot bed is due to a larger pressure drop over the $70 \mathrm{~cm}$ between the measurement positions compared to $15 \mathrm{~cm}$ in the case of the cold bed. It should be noted that no scaling rule is applied in choosing the pressure data positions. However, the positions are such that one is within the grid zone (occupying up to $25 \%$ of the bed height) and the other within the constant density zone. The variation of mean pressure drop and pressure fluctuations at different gas velocities are shown in Figure 4.

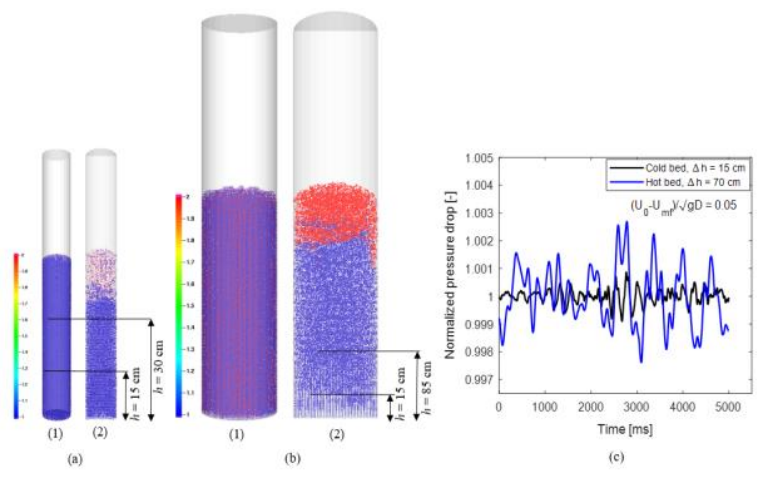

Figure 3. CPFD simulations showing solids distribution (a) cold bed of diameter $10.4 \mathrm{~cm}$, height $50 \mathrm{~cm}$ with (1) initial state (2) final state (b) hot $\left(800^{\circ} \mathrm{C}\right)$ scaled bed of diameter $100 \mathrm{~cm}$, height $270 \mathrm{~cm}$ with (1) initial state (2) final state (c) pressure drop over the indicated positions within the last-5 s of the simulation time. On the colour scale, "1" indicates sand (blue), "2" indicates biomass (red) and number in between indicates the particle mixture.

For the same Froude number, $F r=\left(U_{0}-U_{m f}\right) /$ $\sqrt{g D}$, Figure 4(a) shows that the pressure drop per unit length is close in the two beds. The approximately constant value of the pressure drop at different gas velocities indicates that the bed is well fluidized. Figure 4(b) gives the relative pressure fluctuation computed as $\sigma / p$, where $\sigma$ is the standard deviation and $p$ is the mean fluid pressure over the last $5 \mathrm{~s}$ of the simulation time. The value of $\sigma / p$ increases with increasing gas velocity due to increasing bubble flux. Up to $F r=0.05$, the pressure fluctuations are similar in the two beds, and also the same at both bed positions. At higher Froude numbers, the difference in the values of $\sigma / p$ between the two beds (particularly at the upper position) suggests that slugs flow in the beds (although not clear in the simulations). In the cold bed, the experiments show that slugs begin to flow in the upper bed position (observed at $28.7 \mathrm{~cm}$ above the distributor) when $\mathrm{Fr}=0.078$. The flow of slugs results in higher fluctuations and bed instability.

With the strong agreement in both the simulated and experimental results, it therefore shows that by using the computed scaled bed aspect ratio, the bubble flow behavior in the hot large bed is similar to that in the cold small bed within the bubble regime.

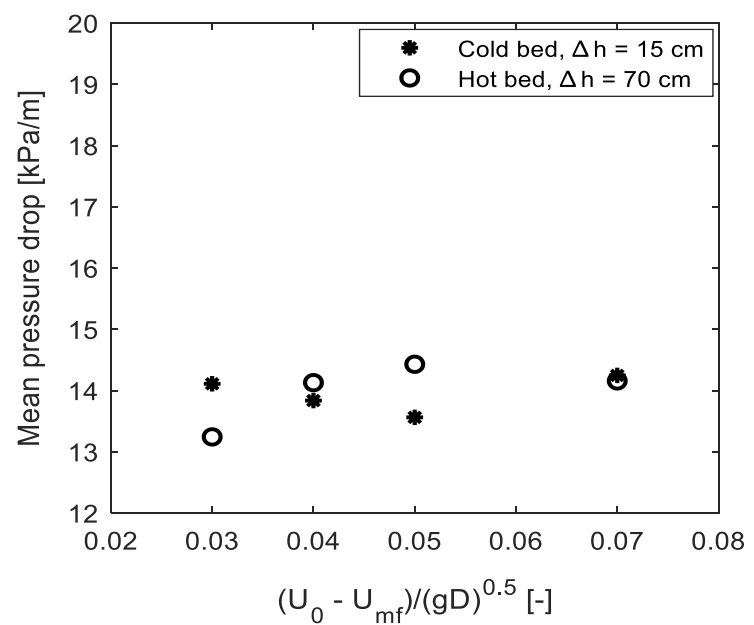

(a)

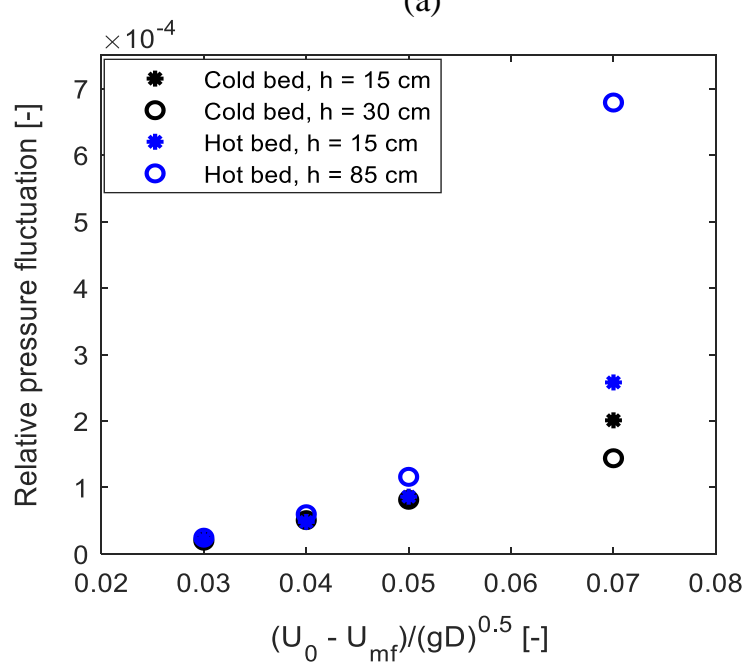

(b)

Figure 4. Comparison between cold bed of diameter 10.4 $\mathrm{cm}$, aspect ratio 5 and hot $\left(800^{\circ} \mathrm{C}\right)$ scaled bed of diameter $100 \mathrm{~cm}$, aspect ratio 2.7 at different values of $\left(U_{0}-\right.$ $\left.U_{m f}\right) / \sqrt{g D}$ (a) mean pressure drop (b) relative fluid pressure fluctuation. 


\section{Conclusions}

In this study, a method for scaling up a cold bed to a hot larger bed was introduced. Using an output such as bed expansion ratio, the proposed scaling approach provides a means of achieving dynamic similarity between two different beds without constraining the fluid and particle properties as well as the bed height, thereby allowing the same properties of fuel particles and bed material type to be used. For illustrations, a $10.4 \mathrm{~cm}$ diameter cold bed containing a mixture of biomass and sand particles was scaled up to a large hot bed of $100 \mathrm{~cm}$ diameter and the results from CPFD model simulations showed that both beds have clear similarity in bubbling behavior.

Beside bed expansion ratio, other bubble properties including the rise velocity and volumetric bubble flux can also be considered in the scaling approach, provided that such properties are well correlated with the bed geometry and particle properties.

Although the new scaling up approach introduced in this work is verified numerically using commercial simulation software, further verifications using experimental data are necessary.

\section{References}

C. E. Agu, L.-A. Tokheim, C. Pfeifer and B. M. E. Moldestad. Behaviour of Biomass Particles in a Bubbling Fluidized Bed: A Comparison between Wood Pellets and Wood Chips. Chemical Engineering Journal, 363: 84-98, 2019(a).

C. E. Agu, A. Ugwu, C. Pfeifer, M. S. Eikeland, L.-A. Tokheim and B. M. E. Moldestad. Investigation of Bubbling Behaviour in Deep Fluidized Beds at Different Gas Velocities using Electrical Capacitance Tomography. Industrial \& Engineering Chemistry Research, 58(5): 2084 2098, 2019(b).

C.E. Agu, L.-A. Tokheim, M. S. Eikeland and B.M.E. Moldestad. Improved models for predicting bubble velocity, bubble frequency and bed expansion in a bubbling fluidized bed. Chemical Engineering Research and Design, 141: 361-371, 2019(c).

C.E. Agu, C. Pfeifer, M. Eikeland, L.-A. Tokheim and B.M.E. Moldestad. Models for predicting average bubble diameter and volumetric bubble flux in deep fluidized beds. Industrial \& Engineering Chemistry Research, 57(7): 26582669,2018

M.J. Andrews and P.J. O'Rourke. The multiphase particle-incell (MP-PIC) method for dense particulate flows. International Journal of Multiphase Flow, 22(2): 379-402, 1996.

M. Biglari, H. Liu, A. Elkamel and A. Lohi. Application of scaling-law and CFD modeling to hydrodynamics of circulating biomass fluidized bed gasifier. Energies, 9(7): 504-518, 2016.

C. Chen, J. Werther, S. Heinrich, H.-Y. Qi and E.-U. Hartge. CPFD simulation of circulating fluidized bed risers. Powder Technology, 235: 238-247, 2013.

J. Chladek, C.K. Jayarathna, B.M.E. Moldestad and L.-A. Tokheim. Fluidized bed classification of particles of different size and density. Chemical Engineering Science, 177: 151-162, 2018.

J. H. Choi, J. E. Son and S. D. Kim. Bubble size and frequency in gas fluidized beds. Journal of Chemical Engineering of Japan, 21(2): 171-178, 1988.

R. C. Darton, R. D. La Naueza, J. F. Davidson and D. Harrison. Bubble growth due to coalescence in fluidized beds. Transactions of the Institution of Chemical Engineers, 55: 274, 1977.

F. Fotovat, R. Ansart, M. Hemati, O. Simonin and J. Chaouki. Sand-assisted fluidization of large cylindrical and spherical biomass particles: Experiments and simulation. Chemical Engineering Science, 126: 543-559, 2015(a).

F. Fotovat, A. Abbasi, R.J. Spiteri, H. de Lasa and J.A. Chaouki. CPFD model for a bubbly biomass-sand fluidized bed. Powder Technology, 275: 39-50, 2015(b).

L. R. Glicksman, M. Hyre and K. Woloshun. Simplified scaling relationships for fluidized beds. Powder Technology, 77(2): 177-199, 1993.

L. R. Glicksman. Scaling relationships for fluidized beds. Chemical Engineering Science, 43(6): 1419-1421, 1988.

L. R. Glicksman. Scaling relationships for fluidized beds. Chemical Engineering Science, 39(9): 1373-1379, 1984.

A. Hepbasli. Estimation of bed expansions in a freelybubbling three-dimensional gas-fluidized bed. International Journal of Energy Research, 22: 1365, 1998.

M. Horio, A. Nonaka, I. Sawa and Y. Muchi. A new similarity rule for fluidized bed scale-up. AIChE Journal, 32(9): 14661482, 1986.

S. Kraft, M. Kuba and H. Hofbauer. The behavior of biomass and char particles in a dual fluidized bed gasification system. Powder Technology 338: 887-897, 2018.

S. Mori and C. Y. Wen. Estimation of bubble diameter in gaseous fluidized beds. AIChE Journal, 21(1): 109-115, 1975.

J. B. Romero and L. N. Johanson. Factors affecting fluidized bed quality. Chemical Engineering Progress. Symposium Series 58: 28, 1962.

P. Salatino and R. Solimene. Mixing and segregation in fluidized bed thermochemical conversion of biomass. Powder Technology 316: 29, 2017.

R. K. Thapa and B. M. Halvorsen. Scaling of bubbling fluidized bed reactors with Glicksman's viscous limit set and CFD simulation. International Journal of Computational Methods and Experimental Measurements 2(2): 135-144, 2014.

C. Y. Wen and Y. H. Yu. A Generalized Method for Predicting the Minimum Fluidization Velocity. AIChE Journal, 12(3): 610-612, 1966(a).

C. Y. Wen and Y. H. Yu. Mechanics of fluidization. Chemical Engineering Progress Symposium Series, 162: 100-111, 1966(b).

J. Werther. Effect of gas distributor on the hydrodynamics of gas fluidized beds. German Chemical Engineering, 1: 166, 1978.

J. Zhang and G. Xu. Scale-up of bubbling fluidized beds with continuous particle flow based on particle-residence-time distribution. Particuology, 19: 155-163, 2015. 